\title{
Porcine-derived acellular dermal collagen matrix and enamel matrix derivative for the treatment of infrabony defect in the esthetic area
}

\author{
Martina Stefanini, Diego Bianchelli, Matteo Sangiorgi \\ Department of Biomedical and Neuromotor Sciences, Bologna University, Bologna 40125, Italy.
}

Correspondence to: Dr. Martina Stefanini, Department of Biomedical and Neuromotor Sciences, Bologna University, Via San Vitale 59, Bologna 40125, Italy. E-mail: martinastefanini3@gmail.com

\begin{abstract}
How to cite this article: Stefanini M, Bianchelli D, Sangiorgi M. Porcine-derived acellular dermal collagen matrix and enamel matrix derivative for the treatment of infrabony defect in the aesthetic area. Plast Aesthet Res 2021;8:15.

http://dx.doi.org/10.20517/2347-9264.2020.186
\end{abstract}

Received: 30 Sep 2020 First Decision: 18 Jan 2021 Revised: 22 Jan 2021 Accepted: 9 Feb 2021 Published: 9 Mar 2021

Academic Editors: Filippo Citterio, Raúl González-García Copy Editor: Xi-Jun Chen Production Editor: Yue-Yue Zhang

\begin{abstract}
This case report describes a modified connective tissue graft wall technique with enamel matrix derivative proposed to treat vertical bony defects. The surgical procedure consisted of a coronally advanced flap with a porcine-derived acellular dermal matrix placed below, acting as a buccal soft tissue wall of the bony defect. The patient showed a bony defect between \#12 and \#13 with horizontal bone loss and a deep infrabony component at the mesial area of the canine. At one year after surgery, the position of the interdental papilla and the clinical attachment level gain were improved along with radiographic bone defect fill.
\end{abstract}

Keywords: Wall technique, coronally advanced flap, connective tissue graft, acellular dermal matrix, infrabony defect, enamel matrix derivatives

\section{INTRODUCTION}

The aim of periodontal regeneration is to improve clinical outcomes of teeth considered to be compromised, namely characterized by the presence of deep pockets and loss of supporting tissues, including alveolar bone, cementum, and periodontal ligament ${ }^{[1]}$. The persistence of deep pockets after a periodontal therapy has been correlated with infrabony defects, posing an elevated risk of future periodontal destruction and, eventually, tooth $\operatorname{loss}^{[2]}$. Teeth presenting deep pockets and diminished periodontal support are considered 
hopeless by many dentists, mostly because of the insufficient periodontal attachment ${ }^{[3-5]}$; therefore, periodontal regenerative procedures would help change this prognosis.

In ideal conditions, regenerative therapy of infrabony defects should achieve a clinical attachment level $(\mathrm{CAL})$ gain equivalent to the probing depth (PD) reduction, while avoiding an increase in gingival recession $(\mathrm{REC})$ as a consequence of the therapy ${ }^{[6]}$.

As we have learned from guided bone regeneration ${ }^{[7]}$, critical factors to achieve successful periodontal regeneration are related to the ability of the surgical procedure to create and maintain adequate space for the blood clot and provide its stability and protection through primary closure ${ }^{[8]}$.

The introduction of papilla preservation techniques has had a positive impact on the stability of the wound and the possibilities of achieving healing by primary intention ${ }^{[9,10]}$. Later on, with the concomitant evolution of biomaterials, minimally invasive and microsurgical approaches have been described.

Cortellini et al. ${ }^{[11,12]}$ suggested the minimally invasive surgical technique (MIST) and a subsequent modification (M-MIST). The background foundation for this technique is the conception of the minimally invasive technique (MIT) introduced in $1995^{[13]}$ : minimal wounds, minimal flap elevation, and gentle manipulation of soft and hard tissues. MIST aims to diminish trauma of the surgery, increase wound stability, allow first-intention healing, reduce the time of the surgery, and minimize patient morbidity.

In $2009^{[14]}$, the single-flap approach (SFA) with guided tissue regeneration (GTR) using a bioresorbable membrane and graft biomaterial was described. This procedure is specifically intended for periodontal regenerative techniques, in intraosseous defects extended predominantly on the oral or buccal aspect. The SFA technique consists in the elevation of the flap to expose the defect only from the buccal or the oral side, keeping the other one untouched. Significant CAL gain and minimal post-surgical recession were reported, thus this technique might be appropriate to treat defects in esthetic areas.

An innovative approach combining papilla preservation technique and coronally advanced flap (CAF) was proposed by Zucchelli and De Sanctis ${ }^{[15]}$ to reduce the amount of soft tissue recession, particularly in the esthetic zone.

In 2014, Zucchelli et al ${ }^{[16]}$ introduced the connective tissue graft wall technique in combination with enamel matrix derivative (EMD) below a CAF, applied in Miller Class IV gingival recessions. The connective tissue graft (CTG) was utilized as a buccal soft tissue wall for the interproximal defect and was involved in a bilaminar technique to achieve a complete coverage of the treated roots. The dense sub-epithelial palatal connective tissue represented a rigid barrier that could prevent the soft tissue from collapsing into the vertical bony defect and thus promote blood clot stabilization.

Recently, the CTG wall technique has been modified for the treatment of deep vertical bony defects, proposing a palatal incision to expose the intrabony component of the defect, in order to reduce buccal and interdental soft tissue recession to a minimum ${ }^{[17]}$. Several advantages can be ascribed to this surgical technique, including the maintenance of the connection between the supracrestal soft tissue, the papilla complex, and the buccal flap, as well as a minimized risk of unesthetic outcomes in the event of non-ideal soft tissue closure. According to the authors, this approach may enhance the complete resolution of deep intrabony defects, while improving the quality of the vestibular and interproximal soft tissues. Additionally, since the CTG turns the defect into a contained defect, it is possible to apply solely amelogenins because there is no need to use additional biomaterials to ensure clot stabilization ${ }^{[18]}$. 


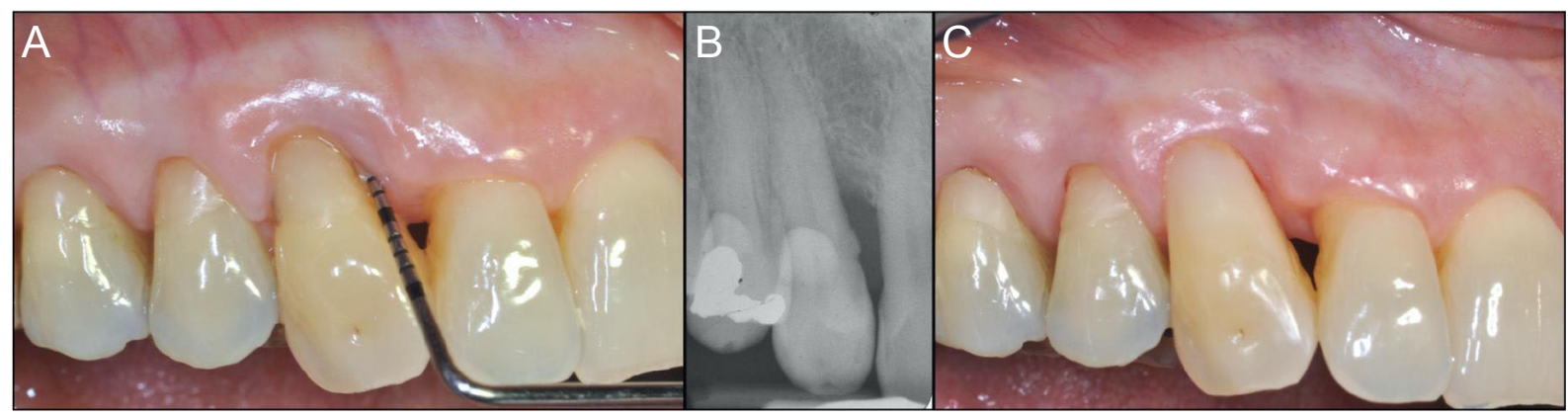

Figure 1 Baseline situation. $(A, C)$ Clinical examination revealed the presence of a recession of the interdental soft tissue situated at the distal line angle of tooth \#12 and at the mesial angle of the canine. (B) The radiographic examination showed a bony defect between $\# 12$ and \#13 with horizontal supracrestal bone loss and a deep infrabony defect at the mesial side of canine.

On the downside, the use of a CTG may result in increased patient discomfort in comparison with techniques requiring a single surgical area. Connective tissue graft substitutes, such as porcine-derived acellular dermal matrix (PADM, Mucoderm, Botiss biomaterials), have been characterized by a rising popularity among periodontists, mainly because of their advantages: reduction of surgical time, limited patient morbidity, and minimized postoperative complications ${ }^{[19]}$.

The following case report describes the treatment of an infrabony defect in the esthetic area with a modified CTG wall technique. A connective tissue substitute was applied beneath the CAF to function as the missing buccal wall of the defect, which was treated with EMD to obtain periodontal regeneration along with an esthetic result.

\section{CASE REPORT}

A 55-year-old woman was referred to the Biomedical and Neuromotor Sciences Department of Bologna University, Bologna, Italy, for a periodontal screening visit. The patient presented clinical signs of inflammation and several teeth with periodontal attachment loss and radiographic infrabony defects [Figure 1] that suggest a diagnosis of periodontitis Stage III Grade $\mathrm{B}^{[20]}$.

While treating the periodontal disease, one of the patient's requests was to improve the esthetic of her smile, complaining of the loss of papilla between \#12 and \#13.

No systemic contraindications for dental treatment were identified in the medical history of the patient.

Clinical examination revealed interdental soft tissue recession between \#12 and \#13 associated with periodontal attachment loss on the lateral incisor, where the distal portion of the cemento-enamel junction was exposed. The canine presented a composite restoration in the cervical region with carious infiltration. Buccal gingival recessions and non-carious cervical lesions were also detected at the level of the premolars.

At baseline, the following clinical parameters were recorded:

Tooth 13

CAL: distal 3, mesial 10, buccal 4, palatal $4(\mathrm{~mm})$.

PD: distal 3, mesial 7, buccal 2, palatal $4(\mathrm{~mm})$.

REC: distal 0, mesial 3, buccal 2, palatal o (mm). 
Tooth 12

CAL: distal 6, mesial 3, buccal 3, palatal 4 (mm).

PD: distal 3, mesial 3, buccal 2, palatal $4(\mathrm{~mm})$.

REC: distal 3, mesial o, buccal 1, palatal o (mm).

The interdental recession between \#12 and \#13, defined as the distance from the tip of the papilla to the contact point, was $3 \mathrm{~mm}$ [Figure $1 \mathrm{~A}$ ].

The radiographic inspection showed horizontal bone loss at the lateral incisor and a vertical defect at the canine with a $6 \mathrm{~mm}$ intrabony component [Figure 1B].

First, scaling and root planing were performed, and the patient received oral hygiene instructions. New composite restorations were performed on the canine and on $\# 14, \# 15$, and $\# 16$ to restore the non-carious cervical lesions up to the level of maximum root coverage $e^{[21]}$.

After this initial phase, the patient showed low full-mouth plaque and bleeding scores (13\% and 10\%, respectively). The objectives of the non-surgical therapy in the target area for the regenerative procedure were to diminish inflammation and avoid recession of the interdental papilla between \#12 and \#13.

Thin ultrasonic tips were used to debride the root surface, minimizing soft tissue trauma and subsequent shrinkage of the supracrestal tissues covering the infrabony defect. The mucogingival/reconstructive surgery was performed one month after the conclusion of the non-surgical therapy in the target area ${ }^{[15]}$.

The regenerative potential of the defect suggested the use of a regenerative surgical technique; possible alternatives in this case were a traditional regenerative surgery using a bone substitute that could stabilize the clot within the non-contained defect or the connective wall technique using a connective tissue graft. The first option could not allow an improvement of the esthetic condition because it did not involve coronal advancement of the flap and soft tissue management. On the other hand, the connective wall technique would require a connective tissue graft associated with higher patient morbidity. Therefore, we decided to introduce a modification of this technique in order to obtain an improvement in esthetics without increasing morbidity.

The implemented surgical technique was the connective tissue graft wall technique ${ }^{[17]}$, replacing the palatal connective tissue graft with an acellular dermal matrix [Figure 2].

A palatal access was used to reach the bony defect and a combination of enamel matrix derivative and a porcine acellular dermal matrix was applied beneath a coronally advanced buccal flap.

Following buccal and palatal local anesthesia, a horizontal split-thickness incision was made at the base of the palatal anatomical papilla covering the defect. This palatal incision was beveled in order to be as apical as possible once the palatal bone was reached, and then the flap elevation proceeded full thickness. With the same split-thickness incisions at the base of the palatal papillae neighboring the defect, a palatal flap was elevated exposing the palatal side of the bony defect completely. The supracrestal soft tissue was detached from the bone crest with a horizontal split-thickness incision and forced to the buccal side below the contact point.

Once the supracrestal soft tissue situated buccally became mobile, the buccal flap was elevated. The design of the buccal flap was similar to the coronally advanced flap described by Zucchelli and De Sanctis ${ }^{[22]}$ to treat multiple gingival recessions, characterized by submarginal split-thickness surgical papillae at the 


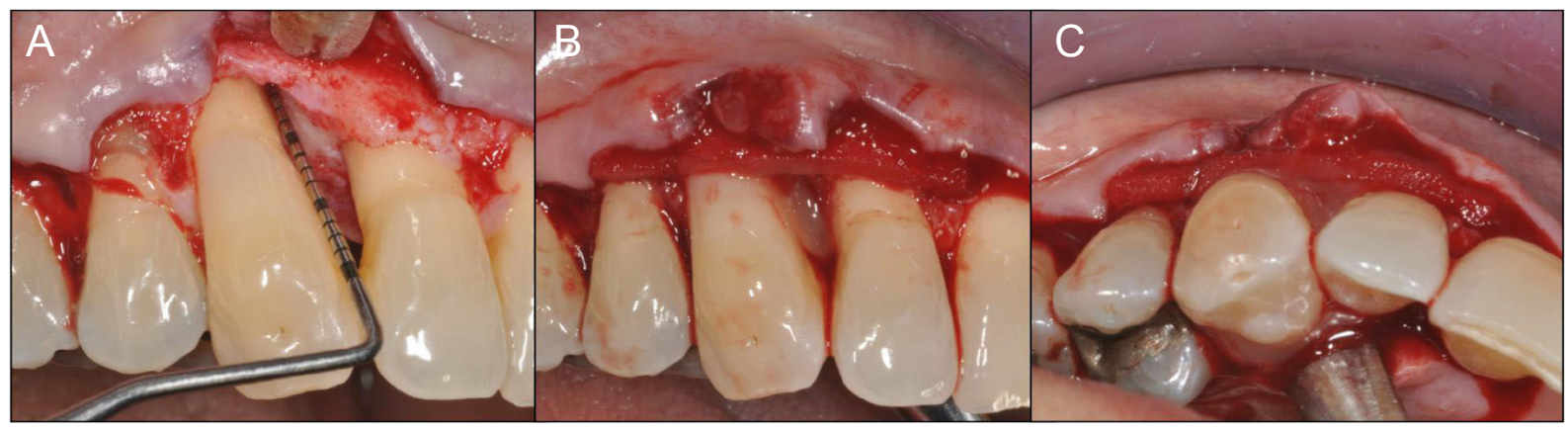

Figure 2 Surgical procedure. (A) The buccal flap design was comparable to the CAF described for the treatment of multiple gingival recessions in mucogingival surgery, characterized by submarginal split-thickness surgical papillae at the interdental area neighboring the defect. Supracrestal soft tissue situated over the bony defect was elevated full thickness. The elevation of the buccal flap proceeded split thickness to enable the coronal advancement of the flap. (B, C) The collagen matrix was sutured at the base of the anatomical papillae of the teeth neighboring the defect, whereas apically it was firmly attached to the periosteum located over the buccal bone apical to the bone defect using single interrupted 7-0 polyglycolic acid sutures. The collagen matrix could act as a buccal missing wall of the defect, and at the same time it could improve root coverage of the neighboring teeth with respect to CAF alone. CAF: Coronally advanced flap.

interdental spaces neighboring the defect. The supracrestal soft tissue covering the bony defect was gently elevated full thickness. Buccal flap elevation proceeded split thickness to allow its coronal advancement. First, the muscle insertions were detached from the periosteum with a deep incision; then, with a more superficial incision, muscle insertions were detached from the inner aspect of the flap. This allowed the supracrestal soft tissues to be passively shifted in a coronal direction, filling the interdental spaces.

De-epithelialization of the buccal aspect of the adjacent anatomical papillae provided connective tissue beds, onto which the surgical papillae of the coronally advanced buccal flap could be sutured. After removal of the granulation tissue located inside the infrabony defect, root planing was performed on the affected teeth. The deepest infrabony component measured $6 \mathrm{~mm}$ [Figure 2A]. The root surfaces were conditioned applying a 24\% EDTA gel for 2 min to remove the smear layer. After a saline rinse, EMD gel was placed on the root surfaces and bony walls and left in place inside the defect.

The acellular dermal collagen matrix was trimmed with a surgical blade in order to have the correct dimensions. Buccally, the matrix had to cover the distance between the mesial healthy papilla and the distal one neighboring the infrabony defect; the apico-coronal dimension was equal to the distance between the cemento-enamel junction of the target tooth and the buccal bone apical to the defect. The collagen matrix was stabilized at the level of the cemento-enamel junction and fixed at the base of the adjacent undetached papillae, acting as a stable barrier over the bone defect lacking a buccal wall.

Thorough hydration of the collagen matrix prior to the application is necessary to increase the flexibility of the matrix, which can be achieved by leaving it in sterile saline solution for $15 \mathrm{~min}$.

The collagen matrix was sutured at the base of the anatomical papillae of the two teeth neighboring the bony defect [Figure $2 \mathrm{~B}$ and $2 \mathrm{C}$ ], whereas apically it was firmly attached to the periosteum above the bone defect using simple interrupted 7-0 polyglycolic acid sutures ${ }^{[23]}$.

The buccal flap was positioned coronally, suturing the surgical papillae to the reciprocal de-epithelialized anatomical papillae with sling sutures around the palatal cingula, similar to the suture technique described for $\mathrm{CAF}$ in the treatment of multiple gingival recessions ${ }^{[22]}$.

The supracrestal soft tissue was forced back to the palatal area beneath the contact point, and then a horizontal 6-0 polyglycolic acid (PGA) internal mattress suture was selected with the purpose of almost 


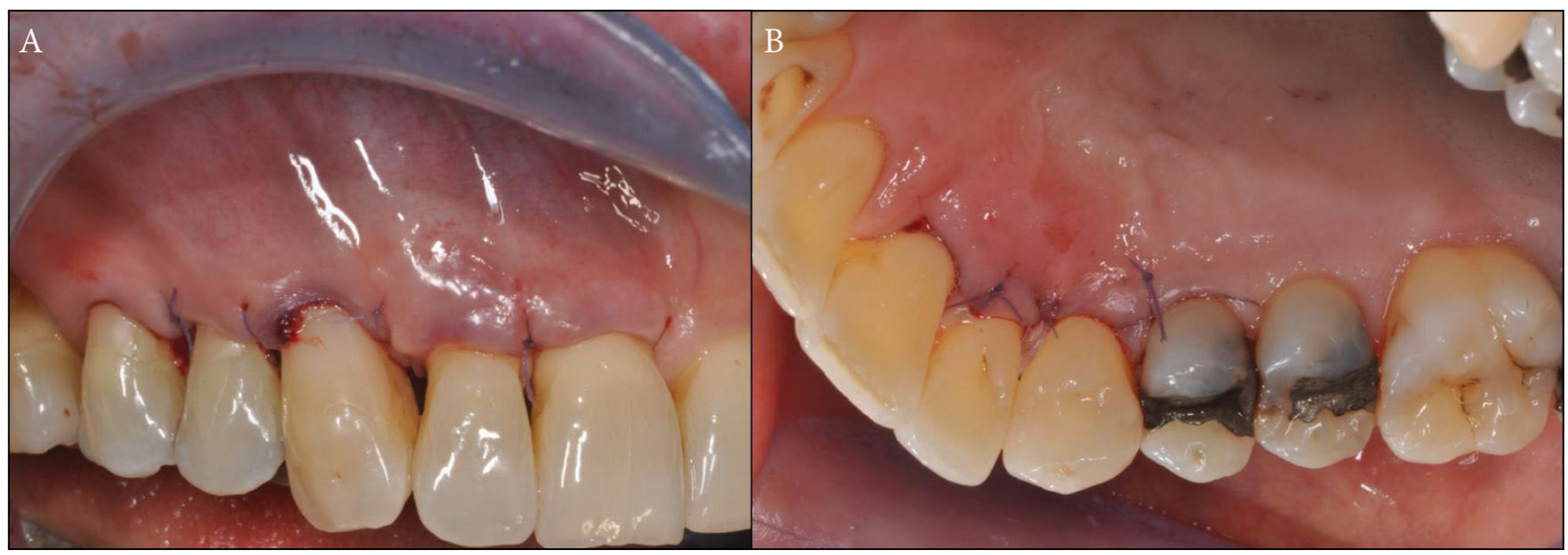

Figure 3. (A, B) A horizontal 6-O PGA internal mattress suture was selected with the purpose of almost connecting the supracrestal soft tissue and the palatal flap, and then a palatal single interrupted 7-O PGA suture achieved healing by first intention between the interdental soft tissue and the palatal flap. PGA: Polyglycolic acid.

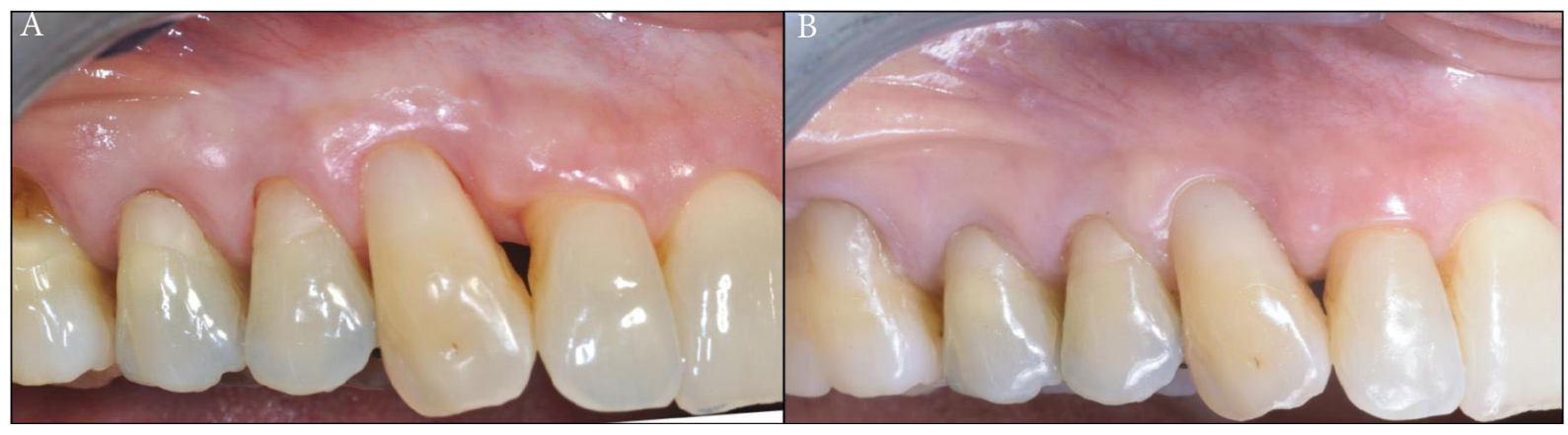

Figure 4 Clinical aspect: at baseline (A); and at 12-month follow-up examination (B). Notice how the interdental soft tissue esthetic has improved and how the papilla between canine and lateral incisor was situated more coronally than the baseline position.

connecting the supracrestal soft tissue and the palatal flap ${ }^{[16]}$. A last palatal simple interrupted 7-0 PGA suture allowed first intention wound closure between the interdental soft tissue and the palatal flap [Figure $3 \mathrm{~A}$ and $3 \mathrm{~B}]$.

For two weeks after the surgery, the patient was asked to rinse with chlorhexidine $0.12 \%$ three times a day. The sutures were removed, and the patient was asked to brush with an ultrasoft post-surgical toothbrush with a roll technique for one month. From the second month the patient used a super-soft toothbrush and from the third month a soft one. The patient was visited for professional tooth cleaning every week for the first three months, and then she went on a three-month recall program.

Twelve months after the surgery, the recession of \#13 was completely covered and the distal line angle of \#12 was partially covered. The tip of the interdental papilla was located more coronal than the baseline position [Figure $4 \mathrm{~B}$ ].

Clinical measures were recorded at 12 months as follows:

\section{Tooth 13}

CAL: distal 4, mesial 4, buccal 2, palatal $4(\mathrm{~mm})$.

PD: distal 4, mesial 4, buccal 2, palatal $4(\mathrm{~mm})$.

REC: distal o, mesial o, buccal o, palatal o ( $\mathrm{mm})$. 


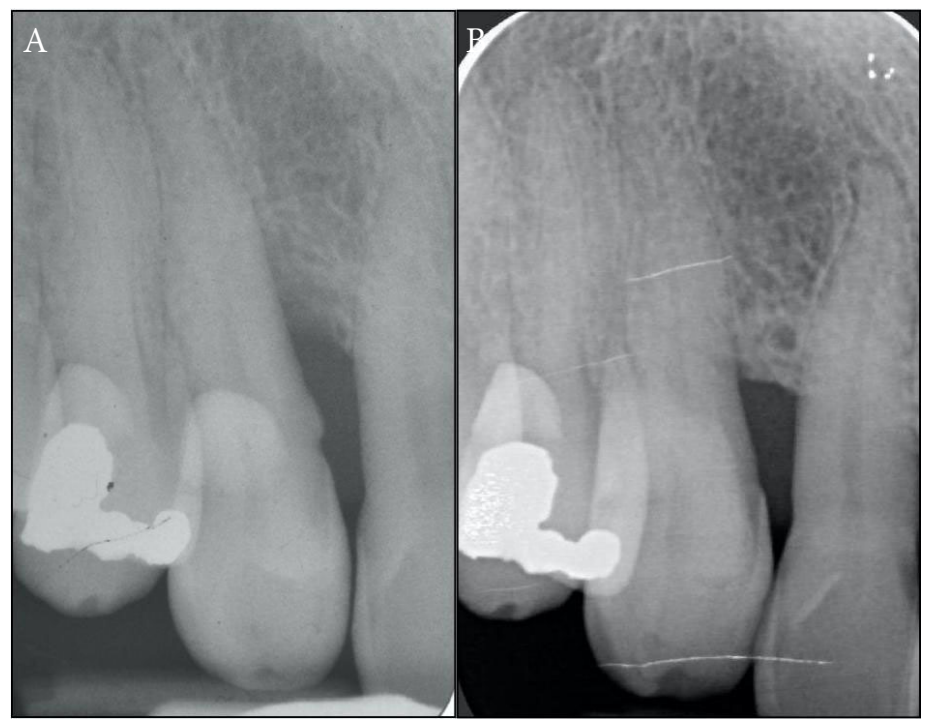

Figure 5. Radiographic examination: at baseline (A); and at 12-month follow-up visit (B). Consider the filling of the deep infrabony defect.

Tooth 12

CAL: distal 4, mesial 3, buccal 2, palatal $4(\mathrm{~mm})$.

PD: distal 3, mesial 3, buccal 2, palatal $4(\mathrm{~mm})$.

REC: distal 1, mesial o, buccal o, palatal o (mm).

The interdental recession between \#12 and \#13, defined as the distance from the tip of the papilla to the contact point, was $1 \mathrm{~mm}$.

Gingival recession coverage was obtained on the adjacent teeth (\#14, \#15\#, and \#16) up to the maximum root coverage level [Figure $4 \mathrm{~B}$ ].

The radiographic inspection showed complete filling of the intrabony defect [Figure 5B].

The patient was extremely pleased with the esthetic outcome at the treated area.

\section{DISCUSSION}

The case presented showed a successful periodontal regeneration along with soft tissue improvement using a PADM instead of the CTG in the wall technique ${ }^{[17]}$ for the treatment of a vertical bony defect in the esthetic area.

One year after the surgery, CAL gain, PD reduction, and complete radiographic fill of the bony defect were achieved, along with the improvement of the buccal and interproximal soft tissue level.

Recently, the CTG wall technique ${ }^{[16,17]}$ has been introduced as a regenerative technique capable of improving the interproximal periodontal parameters in Miller Class IV gingival recessions and in deeper one-wall infrabony defects. According to the technique, an incision is performed at the palate to expose the bony defect, with a split-full-split coronally advanced envelope flap performed on the buccal side and a CTG, obtained from the de-epithelialization of a free gingival graft, sutured coronally at the base of the deepithelialized papillae of the teeth adjacent to the bony defect. According to the authors, this approach may enhance the complete resolution of deep infrabony defects and improve the buccal and interproximal soft 
tissue. Advantages of the CTG include the increase of soft tissue thickness, the limited soft tissue shrinkage of the CAF, and the reduced risk of soft tissue collapse inside the defect ${ }^{[1,24]}$. On the other side, the use of $\mathrm{CTG}^{[25]}$ needs a donor site, which is the palate, usually associated with protracted surgical time, heightened patient morbidity, a greater risk of postoperative complications such as bleeding, excessive sensibility in the donor area, and surgical difficulties in the case of limited palatal depth ${ }^{[26]}$. In the presented case, the main difference from the original wall technique was the application of a PADM instead of the CTG with the aim of overcoming the above-mentioned disadvantages related to the harvesting procedure.

PADM is a collagen matrix extracted from porcine dermis after a specific process with the purpose to remove all the antigenic components ${ }^{[2]}$. The above-mentioned three-dimensional matrix is able to promote the proliferation of fibroblasts, osteoblasts, and endothelial cells, thus encouraging fast revascularization of the entire structure. During the healing process, the collagen matrix is integrated into the surrounding tissue and replaced by the patient's own connective tissue in a natural enzymatic process ${ }^{[2]}$.

One of the most important mechanical properties of the PADM is its ability to regain the initial thickness after compression, withstanding flap pressure. In the presented technique, after the suture, the major segment of the PADM was covering the bony defect, and, thanks to this property, it could be a stable buccal wall, missing in the suprabony and infrabony components of the defect. The PADM wall was able to act as a barrier that prevented the supracrestal soft tissue from collapsing inside the supra- and intrabony components of the defects, turning a non-contained defect into a contained one, where the use of amelogenins without any bone substitutes was indicated [Figure $2 \mathrm{~B}$ and $2 \mathrm{C}$ ]. Moreover, Shirakata et al $^{[27]}$, in a preclinical study, found that the association of CAF, PADM, and EMD obtained more periodontal regeneration on gingival recession defects with respect to the control group without enamel matrix derivative. The authors suggested that PADM may act as an appropriate carrier for enamel matrix derivatives. We can speculate that, in the wall technique, the interaction between PADM and EMD has been a fundamental determining variable influencing cellular behavior in new periodontal regeneration.

The PADM associated with the coronally modified tunnel technique has been successfully used to treat multiple Miller Class I, II, and III gingival recessions ${ }^{[29]}$. The presented case corroborates these results by showing positive results under a CAF regarding root coverage of the gingival defects on the teeth neighboring the bone defect. This can be attributed to the ability of the matrix to incorporate the blood clot, allowing its stability on the root surfaces and therefore favoring the wound healing mechanism that leads to root coverage.

Recently, PADM has been proposed for soft tissue augmentation in the buccal aspect of single implant sites $^{[30]}$, demonstrating that the CAF/PADM combination was effective in the treatment of the previously mentioned situation, gaining an average of $1.2 \pm 0.18 \mathrm{~mm}$ of buccal soft tissue thickness. In the presented case, the improvement of the height of the interdental soft tissues, with an almost complete soft tissue fill of the interproximal area, can be ascribed to the ability of the PADM to increase and preserve the soft tissue volume $e^{[27]}$.

Considering the limit of the present case report, using a PADM below a coronally advanced flap covering the buccal root surfaces and restoring the buccal bone wall of the defect treated with EMD allows: obtaining periodontal regeneration, e.g., CAL gain and radiographic defect fill; improving the position of the buccal soft tissue and the interdental soft tissue covering the intrabony defect; treating buccal gingival recessions at the teeth neighboring the vertical bony defect; improving esthetic outcome; and reducing postoperative patient discomfort.

Further studies including a larger sample size and a control group are advocated to evaluate the efficacy and the long-term stability of this novel approach. 


\section{DECLARATIONS}

\section{Authors' contributions}

Performed the surgery: Stefanini M.

Each author contributed equally to the writing of the article.

\section{Availability of data and materials \\ Not applicable.}

\section{Financial support and sponsorship \\ None}

\section{Conflicts of interest}

All authors declared that there are no conflicts of interest.

\section{Ethical approval and consent to participate}

Not applicable. An informed consent to participate in the study was obtained from the participant.

\section{Consent for publication}

A written informed consent for publication was obtained.

\section{Copyright}

(c) The Author(s) 2021.

\section{REFERENCES}

1. Cortellini P, Tonetti MS. Clinical concepts for regenerative therapy in intrabony defects. Periodontol 2000 2015;68:282-307.

2. Claffey N, Egelberg J. Clinical indicators of probing attachment loss following initial periodontal treatment in advanced periodontitis patients. J Clin Periodontol 1995;22:690-6.

3. Kwok V, Caton JG. Commentary: prognosis revisited: a system for assigning periodontal prognosis. J Periodontol 2007;78:2063-71.

4. Lang NP, Tonetti MS. Periodontal diagnosis in treated periodontitis. Why, when and how to use clinical parameters. $J$ Clin Periodontol 1996;23:240-50.

5. McGuire MK, Nunn ME. Prognosis versus actual outcome. III. The effectiveness of clinical parameters in accurately predicting tooth survival. J Periodontol 1996;67:666-74.

6. Cortellini P, Pini Prato G, Tonetti MS. Periodontal regeneration of human infrabony defects. II. Re-entry procedures and bone measures. $J$ Periodontol 1993;64:261-8.

7. Wang HL, Boyapati L. "PASS" principles for predictable bone regeneration. Implant Dent 2006;15:8-17.

8. Position Paper : Periodontal Regeneration. Journal of Periodontology 2005;76:1601-22.

9. Cortellini P, Prato GP, Tonetti MS. The modified papilla preservation technique. A new surgical approach for interproximal regenerative procedures. J Periodontol 1995;66:261-6.

10. Cortellini P, Prato GP, Tonetti MS. The simplified papilla preservation flap. A novel surgical approach for the management of soft tissues in regenerative procedures. Int J Periodontics Restorative Dent 1999;19:589-99.

11. Cortellini P, Tonetti MS. A minimally invasive surgical technique with an enamel matrix derivative in the regenerative treatment of intrabony defects: a novel approach to limit morbidity. J Clin Periodontol 2007;34:87-93.

12. Cortellini P, Tonetti MS. Improved wound stability with a modified minimally invasive surgical technique in the regenerative treatment of isolated interdental intrabony defects. J Clin Periodontol 2009;36:157-63.

13. Harrel SK, Rees TD. Granulation tissue removal in routine and minimally invasive procedures. Compend Contin Educ Dent $1995 ; 16: 960$, 962, 964 passim.

14. Trombelli L, Farina R, Franceschetti G, Calura G. Single-flap approach with buccal access in periodontal reconstructive procedures. $J$ Periodontol 2009;80:353-60.

15. Zucchelli G, De Sanctis M. A novel approach to minimizing gingival recession in the treatment of vertical bony defects. $J$ Periodontol 2008;79:567-74

16. Zucchelli G, Mazzotti C, Tirone F, Mele M, Bellone P, Mounssif I. The connective tissue graft wall technique and enamel matrix derivative to improve root coverage and clinical attachment levels in Miller Class IV gingival recession. Int J Periodontics Restorative Dent 2014;34:601-9.

17. Zucchelli G, Mounssif I, Marzadori M, Mazzotti C, Felice P, Stefanini M. Connective tissue graft wall technique and enamel matrix 
derivative for the treatment of infrabony defects: case reports. Int J Periodontics Restorative Dent 2017;37:673-81.

18. Miron RJ, Sculean A, Cochran DL, et al. Twenty years of enamel matrix derivative: the past, the present and the future. J Clin Periodontol 2016;43:668-83.

19. McGuire MK, Scheyer ET, Gwaltney C. Commentary: incorporating patient-reported outcomes in periodontal clinical trials. $J$ Periodontol 2014;85:1313-9.

20. Caton JG, Armitage G, Berglundh T, et al. A new classification scheme for periodontal and peri-implant diseases and conditions Introduction and key changes from the 1999 classification. J Clin Periodontol 2018;45 Suppl 20:S1-S8.

21. Zucchelli G, Testori T, De Sanctis M. Clinical and anatomical factors limiting treatment outcomes of gingival recession: a new method to predetermine the line of root coverage. J Periodontol 2006;77:714-21.

22. Zucchelli G, De Sanctis M. Treatment of multiple recession-type defects in patients with esthetic demands. J Periodontol 2000;71:150614.

23. Moraschini V, Calasans-Maia MD, Dias AT, et al. Effectiveness of connective tissue graft substitutes for the treatment of gingival recessions compared with coronally advanced flap: a network meta-analysis. Clin Oral Investig 2020;24:3395-406.

24. Cairo F, Pagliaro U, Nieri M. Treatment of gingival recession with coronally advanced flap procedures: a systematic review. $J$ Clin Periodontol 2008;35:136-62.

25. Cairo F, Barbato L, Tonelli P, Batalocco G, Pagavino G, Nieri M. Xenogeneic collagen matrix versus connective tissue graft for buccal soft tissue augmentation at implant site. A randomized, controlled clinical trial. J Clin Periodontol 2017;44:769-76.

26. Fickl S, Fischer KR, Jockel-Schneider Y, Stappert CF, Schlagenhauf U, Kebschull M. Early wound healing and patient morbidity after single-incision vs. trap-door graft harvesting from the palate--a clinical study. Clin Oral Investig 2014;18:2213-9.

27. Shirakata Y, Sculean A, Shinohara Y, et al. Healing of localized gingival recessions treated with a coronally advanced flap alone or combined with an enamel matrix derivative and a porcine acellular dermal matrix: a preclinical study. Clin Oral Investig 2016;20:1791800 .

28. Rothamel D, Benner M, Fienitz T, et al. Biodegradation pattern and tissue integration of native and cross-linked porcine collagen soft tissue augmentation matrices - an experimental study in the rat. Head Face Med 2014;10:10.

29. Cosgarea R, Juncar R, Arweiler N, Lascu L, Sculean A. Clinical evaluation of a porcine acellular dermal matrix for the treatment of multiple adjacent class I, II, and III gingival recessions using the modified coronally advanced tunnel technique. Quintessence Int 2016;47:739-47.

30. Stefanini M, Rendon A, Zucchelli G. Porcine-derived acellular dermal matrix for buccal soft tissue augmentation at single implant sites: a 1-year follow-up case series. Int J Periodontics Restorative Dent 2020;40:121-8. 